\title{
Effect of 3D Polarization profiles on polarization measurements and colliding beam experiments
}

\author{
W. Fischer and A. Bazilevsky \\ Brookhaven National Laboratory, Upton, NY 11973, USA
}

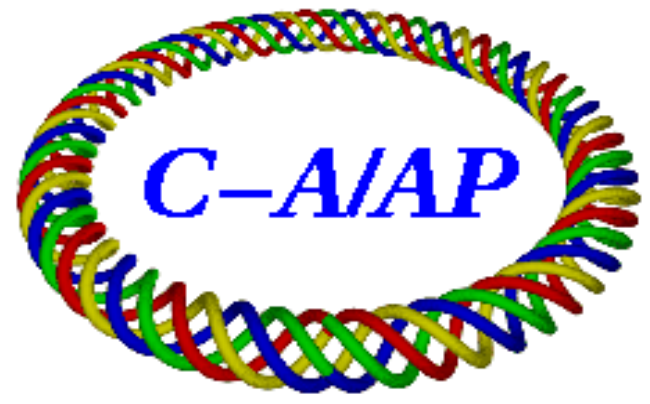

\section{Collider-Accelerator Department Brookhaven National Laboratory Upton, NY 11973}

Notice: This document has been authorized by employees of Brookhaven Science Associates, LLC under Contract No. DE-AC02-98CH10886 with the U.S. Department of Energy. The United States Government retains a nonexclusive, paid-up, irrevocable, world-wide license to publish or reproduce the published form of this document, or allow others to do so, for United States Government purposes. 


\title{
EFFECT OF 3D POLARIZATION PROFILES ON POLARIZATION MEASUREMENTS AND COLLIDING BEAM EXPERIMENTS
}

\author{
Wolfram Fischer* and Alexander Bazilevsky \\ Brookhaven National Laboratory, Upton, NY 11973, USA
}

\section{Abstract}

The development of polarization profiles are the primary reason for the loss of average polarization. Polarization profiles have been parametrized with a Gaussian distribution [1]. We derive the effect of 3-dimensional polarization profiles on the measured polarization in polarimeters, as well as the observed polarization and the figure of merit in single and double spin experiments. Examples from RHIC are provided.

\section{INTRODUCTION}

The Relativistic Heavy Ion Collider (RHIC) is the only collider of spin polarized protons [2]. During beam acceleration and storage profiles of the polarization $P$ develop, which affect the polarization measured in a polarimeter, and the polarization and figure of merit $(F O M)$ in colliding beam experiments. We calculate these for profiles in all dimensions, and give examples for RHIC. Like in RHIC we call the two colliding beams Blue and Yellow. We use the overbar to designate intensity-weighted averages in polarimeters (e.g. $\bar{P}$ ), and angle brackets to designate luminosity-weighted averages in colliding beam experiments (e.g. $\langle P\rangle)$.

\section{COORDINATES}

We use normalized horizontal, vertical, and longitudinal phase space coordinates

$$
\begin{aligned}
\left(x, \tilde{x}^{\prime}\right) & =\left(x, \alpha_{x} x+\beta_{x} x^{\prime}\right) \\
\left(y, \tilde{y}^{\prime}\right) & =\left(y, \alpha_{y} y+\beta_{y} y^{\prime}\right) \\
\left(s, \tilde{s}^{\prime}\right) & =\left(\frac{\phi}{2 \pi} \frac{C}{h}, \frac{C \eta}{2 \pi Q_{s}} \frac{\delta p}{p}\right)
\end{aligned}
$$

where $\left(x, x^{\prime}\right)$ and $\left(y, y^{\prime}\right)$ are the horizontal and vertical phase space coordinates, and $\left(\beta_{x}, \alpha_{x}\right)$ and $\left(\beta_{y}, \alpha_{y}\right)$ the respective lattice functions. $\phi$ is the rf phase, $C$ the circumference, $h$ the harmonic number, $\eta$ the slip factor, $Q_{s}$ the synchrotron tune, and $\delta p / p$ the relative momentum deviation. In the normalized coordinates the linear motion in phase space is represented by a circle on a Poincaré surface of section, and all coordinates have the dimension length.

\footnotetext{
*Wolfram.Fischer@bnl.gov
}

\section{DISTRIBUTIONS}

A Gaussian intensity distribution in phase space is

$$
\begin{aligned}
& I\left(x, \tilde{x}^{\prime}, y, \tilde{y}^{\prime}, s, \tilde{s}^{\prime}\right)= \\
& \frac{N_{b}}{(2 \pi)^{3} \sigma_{x}^{2} \sigma_{y}^{2} \sigma_{s}^{2}} \exp \left\{-\frac{x^{2}+\tilde{x}^{\prime 2}}{2 \sigma_{x}^{2}}-\frac{y^{2}+\tilde{y}^{\prime 2}}{2 \sigma_{y}^{2}}-\frac{s^{2}+\tilde{s}^{\prime 2}}{2 \sigma_{s}^{2}}\right\}
\end{aligned}
$$

where $N_{b}$ is the bunch intensity. We now assume that the spin polarization $P$ can be written as

$$
\begin{aligned}
& P\left(x, \tilde{x}^{\prime}, y, \tilde{y}^{\prime}, s, \tilde{s}^{\prime}\right)= \\
& \quad P_{0} \exp \left\{-\frac{x^{2}+\tilde{x}^{\prime 2}}{2 \sigma_{x, P}^{2}}-\frac{y^{2}+\tilde{y}^{\prime 2}}{2 \sigma_{y, P}^{2}}-\frac{s^{2}+\tilde{s}^{\prime 2}}{2 \sigma_{s, P}^{2}}\right\} .
\end{aligned}
$$

With this dependence the polarization is a function of the normalized horizontal $\left(\sqrt{x^{2}+\tilde{x}^{\prime 2}}\right)$, vertical $\left(\sqrt{y^{2}+\tilde{y}^{\prime 2}}\right)$ and longitudinal $\left(\sqrt{s^{2}+\tilde{s}^{\prime 2}}\right)$ betatron amplitudes. The maximum polarization $P_{0}$ is reached for zero amplitudes in all dimensions. We also introduce the quantities

$$
R_{x}:=\frac{\sigma_{x}^{2}}{\sigma_{x, P}^{2}}, \quad R_{y}:=\frac{\sigma_{y}^{2}}{\sigma_{y, P}^{2}}, \quad \text { and } \quad R_{s}:=\frac{\sigma_{s}^{2}}{\sigma_{s, P}^{2}}
$$

that parametrize the polarization profile, and with which Eq. (3) can be written as

$$
\begin{aligned}
& P\left(x, \tilde{x}^{\prime}, y, \tilde{y}^{\prime}, s, \tilde{s}^{\prime}\right)= \\
& P_{0} \exp \left\{-R_{x} \frac{x^{2}+\tilde{x}^{\prime 2}}{2 \sigma_{x}^{2}}-R_{y} \frac{y^{2}+\tilde{y}^{\prime 2}}{2 \sigma_{y}^{2}}-R_{s} \frac{s^{2}+\tilde{s}^{\prime 2}}{2 \sigma_{s}^{2}}\right\} .
\end{aligned}
$$

Without any polarization profiles we have $\sigma_{x, P} \rightarrow \infty$, $\sigma_{y, P} \rightarrow \infty, \sigma_{s, P} \rightarrow \infty$, and $R_{x}=R_{y}=R_{s}=0$.

\section{POLARIZATION MEASUREMENTS}

The average polarization over all particles, as measured by a $\mathrm{H}$-jet polarimeter $[3,4]$, is

$$
\begin{aligned}
\bar{P} & =\frac{\int_{-\infty}^{+\infty} \stackrel{\int^{\infty}}{+\infty} d x d \tilde{x}^{\prime} d y d \tilde{y}^{\prime} d s d \tilde{s}^{\prime} P(x, \ldots) I(x, \ldots)}{\int \cdots \int d x d \tilde{x}^{\prime} d y d \tilde{y}^{\prime} d s d \tilde{s}^{\prime} I(x, \ldots)} \\
& =\frac{P_{0}}{\left(1+R_{x}\right)\left(1+R_{y}\right)\left(1+R_{s}\right)} .
\end{aligned}
$$


Here and in the following the overbar denotes the intensityweighted average. In a horizontal profile measurement with a thin vertical target [5] we have

$$
\begin{aligned}
\overline{P_{x}}(x) & =\frac{\int_{-\infty}^{+\infty} \cdots \int d \tilde{x}^{\prime} d y d \tilde{y}^{\prime} d s d \tilde{s}^{\prime} P(x, \ldots) I(x, \ldots)}{\int_{-\infty}^{+\infty} d \tilde{x}^{\prime} d y d \tilde{y}^{\prime} d s d \tilde{s}^{\prime} I(x, \ldots)} \\
= & \frac{P_{0}}{\sqrt{1+R_{x}}\left(1+R_{y}\right)\left(1+R_{s}\right)} \exp \left\{-\frac{R_{x} x^{2}}{2 \sigma_{x}^{2}}\right\}
\end{aligned}
$$

Similarly, we have for a vertical profile measurement with a thin horizontal target

$$
\overline{P_{y}}(y)=\frac{P_{0}}{\left(1+R_{x}\right) \sqrt{1+R_{y}}\left(1+R_{s}\right)} \exp \left\{-\frac{R_{y} y^{2}}{2 \sigma_{y}^{2}}\right\},
$$

and for a longitudinal profile measurement

$$
\overline{P_{s}}(s)=\frac{P_{0}}{\left(1+R_{x}\right)\left(1+R_{y}\right) \sqrt{1+R_{s}}} \exp \left\{-\frac{R_{s} s^{2}}{2 \sigma_{s}^{2}}\right\} .
$$

A longitudinal profile can be obtained through timebinning in a $\mathrm{H}$-jet polarimeter [3, 4] or CNI polarimeter [5] measurement.

\section{LUMINOSITY}

For the following we recall the luminosity formula [6-8]

$$
\begin{aligned}
\mathcal{L}= & f_{c} \int_{-\infty}^{+\infty} \ldots \int d x d y d s d t \\
& \times \hat{I}_{B}(x, \ldots, t) \hat{I}_{Y}(x, \ldots, t) \\
& \times \sqrt{\left(\vec{v}_{B}-\vec{v}_{Y}\right)^{2}-\frac{\left(\vec{v}_{B} \times \vec{v}_{Y}\right)^{2}}{c^{2}}}
\end{aligned}
$$

where $f_{c}$ is the bunch collision frequency, and the subscripts $B$ and $Y$ describe quantities of the Blue and Yellow beams respectively. Note that the distributions $\hat{I}$ are only 3-dimensional and also time dependent,

$$
\begin{aligned}
& \hat{I}(x, y, s, t)=\int \cdots \int d \tilde{x}^{\prime} d \tilde{y}^{\prime} d \tilde{s}^{\prime} I(x, \ldots, t) \\
& \quad=\frac{N_{b}}{(2 \pi)^{3 / 2} \sigma_{x} \sigma_{y} \sigma_{s}} \exp \left\{-\frac{x^{2}}{2 \sigma_{x}^{2}}-\frac{y^{2}}{2 \sigma_{y}^{2}}-\frac{s^{2}}{2 \sigma_{s}^{2}}\right\} .
\end{aligned}
$$

$\vec{v}$ is the common velocity of particles in the bunch, and $c$ the speed of light. The rms beam sizes $\sigma_{x, y, s}$ are functions of the time $t$. With neither transverse offset nor crossing angle the luminosity can be written as $[6,9]$

$$
\mathcal{L}=\frac{f_{c} N_{b, B} N_{b, Y}}{2 \pi \sqrt{\left(\sigma_{x, B}^{* 2}+\sigma_{x, Y}^{* 2}\right)\left(\sigma_{y, B}^{* 2}+\sigma_{y, Y}^{* 2}\right)}} h\left(t_{x}, t_{y}\right)
$$

where the superscript ${ }^{*}$ denotes quantities at the interaction point, and the function $h\left(t_{x}, t_{y}\right)$ is the hourglass factor

$$
h\left(t_{x}, t_{y}\right)=\int_{-\infty}^{\infty} \frac{d t}{\sqrt{\pi}} \frac{\exp \left(-t^{2}\right)}{\sqrt{\left(1+t^{2} / t_{x}^{2}\right)\left(1+t^{2} / t_{y}^{2}\right)}}
$$

with

$$
t_{x}^{2}=\frac{2\left(\sigma_{x, B}^{* 2}+\sigma_{x, Y}^{* 2}\right)}{\left(\sigma_{s, B}^{2}+\sigma_{s, Y}^{2}\right)\left(\sigma_{x, B}^{* 2} / \beta_{x, B}^{* 2}+\sigma_{x, Y}^{* 2} / \beta_{x, Y}^{* 2}\right)} .
$$

A similar expression holds for $t_{y}^{2}$.

\section{AVERAGE POLARIZATIONS AND FIGURES OF MERIT IN COLLIDING BEAM EXPERIMENTS}

Let us introduce polarization moments for two colliding beams as

$$
M_{m n}=\left\langle P_{B}^{m} \cdot P_{Y}^{n}\right\rangle,
$$

where $m$ and $n$ are non-negative integers and the angle brackets indicates the luminosity-weighted average over the polarization function.

Important quantities for a collider experiment are average polarizations and figures of merit. For single spin measurements with the Blue and Yellow beams respectively these can be expressed through the polarization moments $M_{m n}$ as

$$
\left\langle P_{B}\right\rangle \equiv M_{10} \quad F O M_{B} \equiv \mathcal{L}\left\langle P_{B}^{2}\right\rangle=\mathcal{L} M_{20}
$$

and

$$
\left\langle P_{Y}\right\rangle \equiv M_{01} \quad F O M_{Y} \equiv \mathcal{L}\left\langle P_{Y}^{2}\right\rangle=\mathcal{L} M_{02}
$$

For double spin experiments we have

$$
\left\langle P_{B} \cdot P_{Y}\right\rangle \equiv M_{11} \quad F O M_{B Y} \equiv \mathcal{L}\left\langle P_{B}^{2} \cdot P_{Y}^{2}\right\rangle=\mathcal{L} M_{22} .
$$

Polarizations, if not equal to 1 , dilute the measured asymmetries and rescaling is needed to get the physics asymmetries. Statistical uncertainties in the measurement scale as $1 / \sqrt{F O M}$, so figures of merit describe the experimental sensitivity or resolution.

We now calculate the moments $M_{m n}$ using the luminosity formulas in the previous section. With Eq. (15) we have

$$
\begin{aligned}
M_{m n}= & \frac{f_{c}}{\mathcal{L}} \int_{-\infty}^{+\infty} \ldots \int d x d y d s d t \\
& \times \hat{I}_{B}(x, \ldots, t) \hat{I}_{Y}(x, \ldots, t) \\
& \times \hat{P}_{B}^{m}(x, \ldots, t) \hat{P}_{Y}^{n}(x, \ldots, t) \\
& \times \sqrt{\left(\vec{v}_{B}-\vec{v}_{Y}\right)^{2}-\frac{\left(\vec{v}_{B} \times \vec{v}_{Y}\right)^{2}}{c^{2}}}
\end{aligned}
$$


where the time-dependent polarization functions in 3 spacial dimensions $\hat{P}^{k}(k=m, n)$ are given by

$$
\begin{aligned}
\hat{P}^{k}(x, y, s, t)= & \frac{\int_{-\infty}^{+\infty} d \tilde{x}^{\prime} d \tilde{y}^{\prime} d \tilde{s}^{\prime} P^{k}(x, \ldots, t) I(x, \ldots, t)}{\int_{-\infty}^{+\infty} d \tilde{x}^{\prime} d \tilde{y}^{\prime} d \tilde{s}^{\prime} I(x, \ldots, t)} \\
= & \frac{P_{0}^{k}}{\sqrt{\left(1+k R_{x}\right)\left(1+k R_{y}\right)\left(1+k R_{s}\right)}} \\
& \times \exp \left\{-\frac{k R_{x} x^{2}}{2 \sigma_{x}^{2}}-\frac{k R_{y} y^{2}}{2 \sigma_{y}^{2}}-\frac{k R_{s} s^{2}}{2 \sigma_{s}^{2}}\right\} .
\end{aligned}
$$

Equation (19) can be expressed as

$$
\begin{aligned}
& M_{m n}=\frac{P_{0, B}^{m} P_{0, Y}^{n}}{\mathcal{L}} \prod_{i=x, y, s} \frac{1}{\left(1+m R_{i, B}\right)\left(1+n R_{i, Y}\right)} \\
& \times f_{c} \int \ldots \int d x d y d s d t \\
& \times \frac{N_{b, B} N_{b, Y}}{(2 \pi)^{3}} \prod_{i=x, y, s} \frac{\sqrt{\left(1+m R_{i, B}\right)\left(1+n R_{i, Y}\right)}}{\sigma_{i, B} \sigma_{i, Y}} \\
& \times \prod_{i=x, y, s} \exp \left\{-\frac{\left(1+m R_{i, B}\right) i^{2}}{2 \sigma_{i, B}^{2}}-\frac{\left(1+n R_{i, Y}\right) i^{2}}{2 \sigma_{i, Y}^{2}}\right\} \\
& \times \sqrt{\left(\vec{v}_{B}-\vec{v}_{Y}\right)^{2}-\frac{\left(\vec{v}_{B} \times \vec{v}_{Y}\right)^{2}}{c^{2}}}
\end{aligned}
$$

The last 4 lines of the above expression have the same form as Eq. (10). The solution of Eq. (12) can be used with the replacements

$$
\begin{aligned}
& \sigma_{i, B}^{* 2} \rightarrow \sigma_{i, B}^{* 2} /\left(1+m R_{i, B}\right) \\
& \sigma_{i, Y}^{* 2} \rightarrow \sigma_{i, Y}^{* 2} /\left(1+n R_{i, Y}\right)
\end{aligned}
$$

for $i=x, y$ and

$$
\begin{aligned}
\sigma_{s, B}^{2} & \rightarrow \sigma_{s, B}^{2} /\left(1+m R_{s, B}\right) \\
\sigma_{s, Y}^{2} & \rightarrow \sigma_{s, Y}^{2} /\left(1+n R_{s, Y}\right) .
\end{aligned}
$$

We obtain

$$
\begin{aligned}
& M_{m n}=P_{0, B}^{m} P_{0, Y}^{n} \prod_{i=x, y, s} \frac{1}{\left(1+m R_{i, B}\right)\left(1+n R_{i, Y}\right)} \\
& \times \frac{\sqrt{\left(\sigma_{x, B}^{* 2}+\sigma_{x, Y}^{* 2}\right)\left(\sigma_{y, B}^{* 2}+\sigma_{y, Y}^{* 2}\right)}}{\sqrt{\left(\frac{\sigma_{x, B}^{* 2}}{1+m R_{x, B}}+\frac{\sigma_{x, Y}^{* 2}}{1+n R_{x, Y}}\right)\left(\frac{\sigma_{y, B}^{* 2}}{1+m R_{y, B}}+\frac{\sigma_{y, Y}^{* 2}}{1+n R_{y, Y}}\right)}} \\
& \times \frac{h\left(t_{x, m n}, t_{y, m n}\right)}{h\left(t_{x}, t_{y}\right)}
\end{aligned}
$$

with

$$
\begin{aligned}
t_{x, m n}^{2}= & \frac{2}{\left(\frac{\sigma_{s, B}^{2}}{1+m R_{s, B}}+\frac{\sigma_{s, Y}^{2}}{1+n R_{s, Y}}\right)} \\
& \times \frac{\left(\frac{\sigma_{x, B}^{* 2}}{1+m R_{x, B}}+\frac{\sigma_{x, Y}^{* 2}}{1+n R_{x, Y}}\right)}{\left(\frac{\sigma_{x, B}^{* 2}}{\left(1+m R_{x, B}\right) \beta_{x, B}^{* 2}}+\frac{\sigma_{x, Y}^{* 2}}{\left(1+n R_{x, Y}\right) \beta_{x, Y}^{* 2}}\right)}
\end{aligned}
$$

and a similar expressions for $t_{y, m n}^{2}$.

Note that the polarizations observed by colliding beam experiments (Eqs. (16) and (17) with the solution of Eq. (24)) generally differ from the average polarization measured by the H-jet polarimeter (Eq. (6)).

\section{SIMPLIFIED CASE}

To simplify the general solution of Eq. (24) considerably we make the following assumptions:

- short bunches, i.e. no hourglass effect

$$
\sigma_{s, B}, \sigma_{s, Y} \ll \beta_{x, B}^{*}, \beta_{y, B}^{*}, \beta_{x, Y}^{*}, \beta_{y, Y}^{*} \Rightarrow h\left(t_{x}, t_{y}\right)=1
$$

- no longitudinal polarization profile

$$
R_{s, B}=R_{s, Y}=0 \Rightarrow h\left(t_{x, m n}, t_{y, m n}\right)=1
$$

- equal transverse polarization profiles in both beams, $P_{0, B}=P_{0, Y}=P_{0}$;

$$
R_{x, B}=R_{x, Y}=R_{x} ; R_{y, B}=R_{y, Y}=R_{y}
$$

- round beams of the same size in both rings,

$$
\sigma_{x, B}^{*}=\sigma_{y, B}^{*}=\sigma_{x, Y}^{*}=\sigma_{y, Y}^{*}=\sigma^{*}
$$

Equation (24) can then be written as

$$
\begin{aligned}
& M_{m n}=\frac{P_{0}^{m+n}}{\sqrt{\left(1+\frac{m+n}{2} R_{x}\right)\left(1+\frac{m+n}{2} R_{y}\right)}} \\
& \quad \times \frac{1}{\sqrt{\left(1+m R_{x}\right)\left(1+n R_{x}\right)\left(1+m R_{y}\right)\left(1+n R_{y}\right)}}
\end{aligned}
$$

and the cases of Eqs. (16), (17) and (18) become

$$
\begin{aligned}
\left\langle P_{B}\right\rangle \text { or }\left\langle P_{Y}\right\rangle= & \frac{P_{0}}{\left[\left(1+\frac{1}{2} R_{x}\right)\left(1+\frac{1}{2} R_{y}\right)\right]^{1 / 2}} \\
& \times \frac{1}{\left[\left(1+R_{x}\right)\left(1+R_{y}\right)\right]^{1 / 2}},
\end{aligned}
$$

$$
\begin{aligned}
\left\langle P_{B}^{2}\right\rangle \text { or }\left\langle P_{Y}^{2}\right\rangle= & \frac{P_{0}^{2}}{\left[\left(1+R_{x}\right)\left(1+R_{Y}\right)\right]^{1 / 2}} \\
& \times \frac{1}{\left[\left(1+2 R_{x}\right)\left(1+2 R_{y}\right)\right]^{1 / 2}}, \\
\left\langle P_{B} \cdot P_{Y}\right\rangle & =\frac{P_{0}^{2}}{\left[\left(1+R_{x}\right)\left(1+R_{y}\right)\right]^{3 / 2}}, \\
\left\langle P_{B}^{2} \cdot P_{Y}^{2}\right\rangle & =\frac{P_{0}^{4}}{\left[\left(1+2 R_{x}\right)\left(1+2 R_{y}\right)\right]^{3 / 2}} .
\end{aligned}
$$


The ratio between the polarization $\bar{P}$ measured by the $\mathrm{H}$-jet and the polarization $\langle P\rangle$ observed in a single spin colliding beam experiment is for the simplified case

$$
\begin{aligned}
\frac{\overline{P_{B}}}{\left\langle P_{B}\right\rangle} \text { or } \frac{\overline{P_{Y}}}{\left\langle P_{Y}\right\rangle} & =\sqrt{\frac{\left(1+\frac{1}{2} R_{x}\right)\left(1+\frac{1}{2} R_{y}\right)}{\left(1+R_{x}\right)\left(1+R_{y}\right)}} \\
& \approx 1-\frac{R_{x}+R_{y}}{4} \text { for } R_{x}, R_{y} \ll 1 .
\end{aligned}
$$

\section{EXAMPLES FROM RHIC}

For illustration we take the RHIC $250 \mathrm{GeV}$ polarized proton run in 2011 [10], with $\beta_{x, y}^{*}=0.6 \mathrm{~m}$ at Interaction Point 8 (PHENIX) and $\sigma_{s}=0.6 \mathrm{~m}$ in both rings.

We consider the two cases of $R_{x}=0$ and $R_{x}=R_{y}$, both with equal polarization profiles in both beams. The former case is expected if all machines in the acceleration chain are perfectly flat, and the horizontal and vertical planes decoupled. The latter case is expected for fully coupled machines. Profile parameters of $R_{x} \approx R_{y} \approx 0.2$ were observed at $250 \mathrm{GeV}$ in Run-11 [11]. The longitudinal profile parameter $R_{s}$ is not yet determined for Run-11 but was found to be small in Run-9.

Figure 1 (a) shows the relative reduction (i.e. relative to the case without polarization profiles) of the average polarization $\bar{P}$ measured by an $\mathrm{H}$-jet as a function of the vertical profile parameter $R_{y}$. The two cases $R_{x}=0$ and $R_{x}=R_{y}$ are shown for $R_{s}=0,0.01,0.05,0.1$ each. Figure 1 (b) displays the ratio of the polarization measured by an $\mathrm{H}$-jet to the polarization seen in a single spin colliding beam experiment.

Figure 2 exhibits the effect of the polarization profiles on the average polarization and figure of merit in single spin colliding beam experiments. Figure 3 shows the effect in double spin experiments.

\section{SUMMARY}

The development of polarization profiles are the main mechanism for the reduction of the average polarization in accelerators, i.e. a reduction compared to the central value $P_{0}$. In the case of RHIC polarization profiles have an considerable impact on both the average beam polarization and the figures of merit in colliding beam experiments, with typical $R$-values of about 0.2 in both transverse planes. Because of the profiles the polarization measured by polarimeters is different from the polarization seen by the experiments, and corrections depend on profiles in all three planes. For precision spin experiments polarimetry must provide measurements of the $R$-values.

\section{ACKNOWLEDGMENTS}

The authors are thankful for discussion to the members of the RHIC Spin Collaboration, in particular E. Aschenauer, H. Huang, A. Poblaguev, T. Roser, B. Schmidke, D. Smirnov, and A. Zelenski.
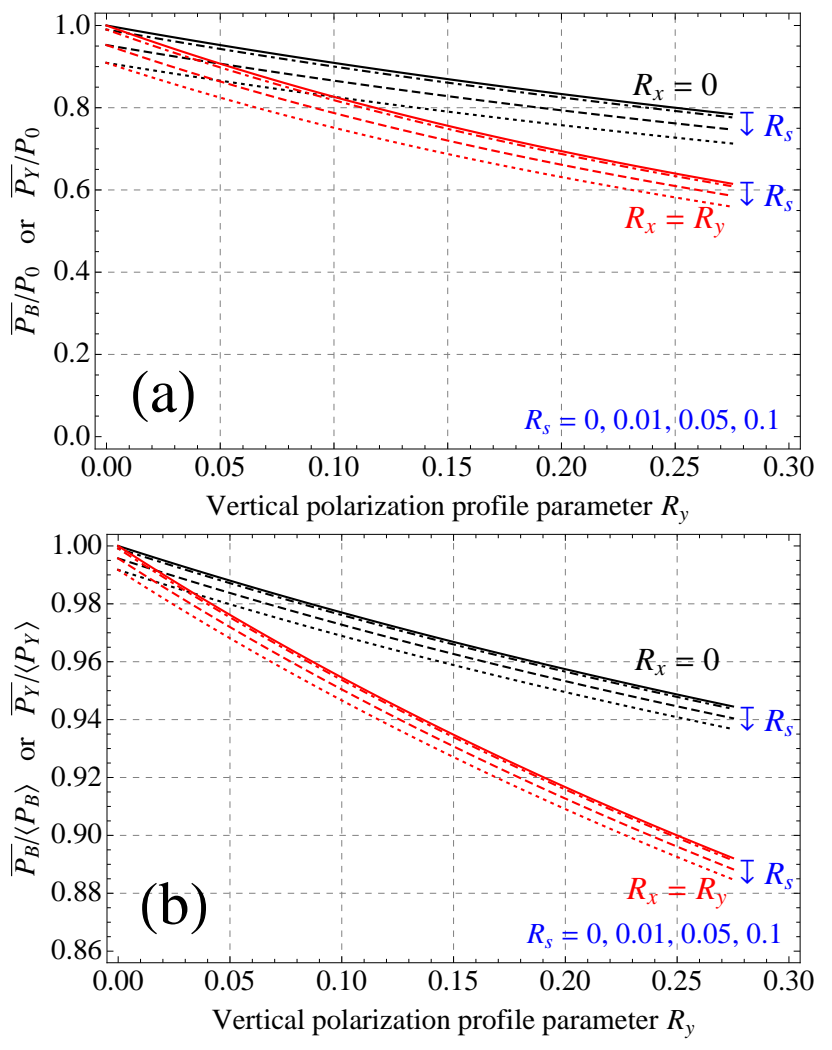

Figure 1: (a) Relative reduction of the average polarization, $\overline{P_{B}} / P_{0}$ or $\overline{P_{Y}} / P_{0}$, as a function of the vertical profile parameter $R_{y}$. (b) Ratio of the polarization measured by an $\mathrm{H}$-jet to the average polarization in single spin colliding beam experiments, $\overline{P_{B}} /\left\langle P_{B}\right\rangle$ or $\overline{P_{Y}} /\left\langle P_{Y}\right\rangle$. The cases $R_{x}=0$ and $R_{x}=R_{y}$ are shown for $R_{s}=$ $0,0.01,0.05,0.1$ each.

Work supported by Brookhaven Science Associates, LLC under Contract No. DE-AC02-98CH10886 with the U.S. Department of Energy.

\section{REFERENCES}

[1] A. Bazilevsky, "Polarization profile and its impact on polarization measurement", presentation at XII ${ }^{\text {th }}$ International Workshop on Polarized Sources, Targets, and Polarimetry PSPT2007, New York (2007).

[2] T. Roser, W.W. MacKay et al., "Configuration manual, polarized proton collider at RHIC”, Rev. 2 (2006).

[3] A. Zelenski et al., "Polarized $\mathrm{H}^{-}$-jet polarimeter for absolute proton polarization measurements in RHIC", proceedings SPIN 2002, AIP Conference Proceedings 675, pp. 954962 (2002).

[4] T. Wise et al., "Polarized hydrogen jet target for measurement of RHIC proton beam polarization", proceedings SPIN 2004, World Scientific, pp. 757-760 (2004).

[5] H. Huang and K. Kurita, "Fiddling Carbon Strings with Polarized Proton Beams", Beam Instrumentation Workshop 

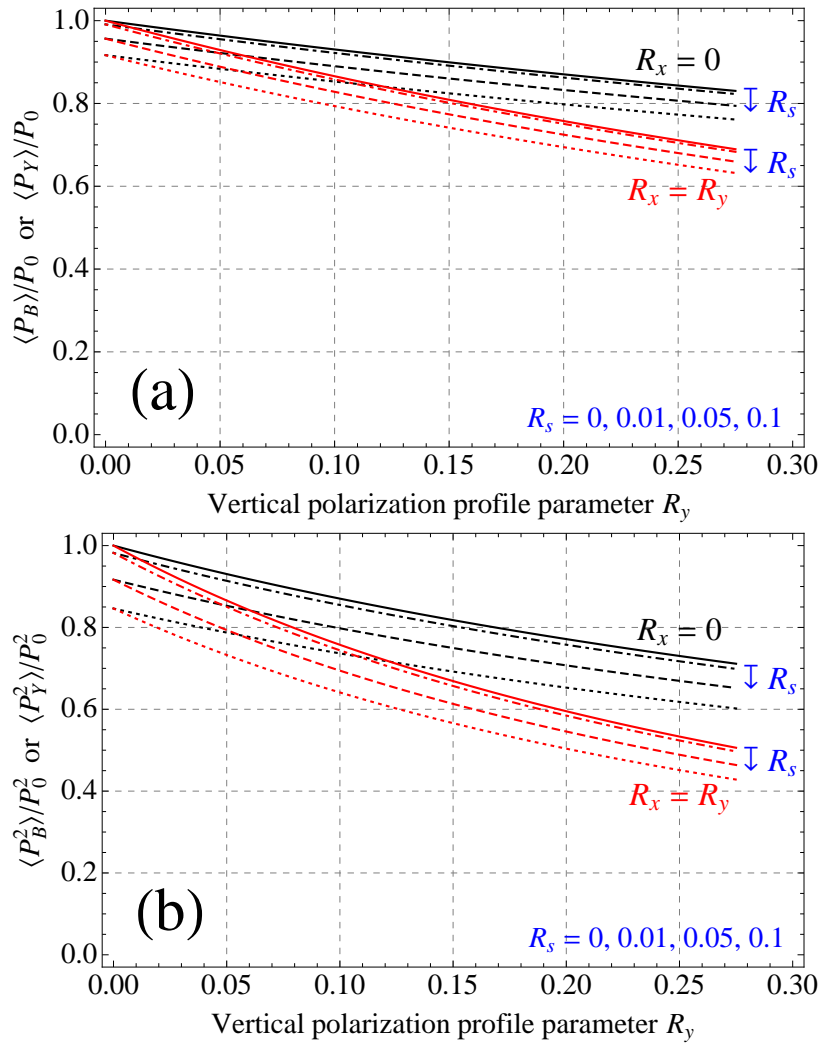

Figure 2: For single spin colliding beam experiments (a) relative reduction of the average polarization $\left\langle P_{B}\right\rangle / P_{0}$ or $\left\langle P_{Y}\right\rangle / P_{0}$, and (b) relative reduction of the figure of merit $\left\langle P_{B}^{2}\right\rangle / P_{0}^{2}$ or $\left\langle P_{Y}^{2}\right\rangle / P_{0}^{2}$, as a function of the vertical profile parameter $R_{y}$. The cases $R_{x}=0$ and $R_{x}=R_{y}$ are shown for $R_{s}=0,0.01,0.05,0.1$ each.

2006 (BIW06), Batavia, IL, May 1-4, 2006, AIP Conference Proceedings 868, pp. 3-21 (2006).

[6] M.A. Furman and M.S. Zisman, "Luminosity", in "Handbook of accelerator physics and engineering", (A.W. Chao and M. Tigner eds.), World Scientific (1999).

[7] M. Conte, W.W. MacKay, "An introduction to the physics of particle accelerators", 2nd ed., World Scientific (2008).

[8] W.W. MacKay, "Luminosity as calculated from machine parameters", BNL C-A/AP/89 (2002).

[9] M.A. Furman, PAC'91, pp. 422-424 (1991). "Hourglass effect for asymmetric colliders", proceedings Particle Accelerator Conference 1991, San Francisco, pp. 422-424 (1991)

[10] H. Huang et al., ' 'RHIC polarized proton operation” proceedings Particle Accelerator Conference 2011, New York, pp. 41-45 (2011).

[11] H. Huang (editor), Minutes CAD spin meetings, www.rhichome.bnl.gov/AP/spinmeeting.htm
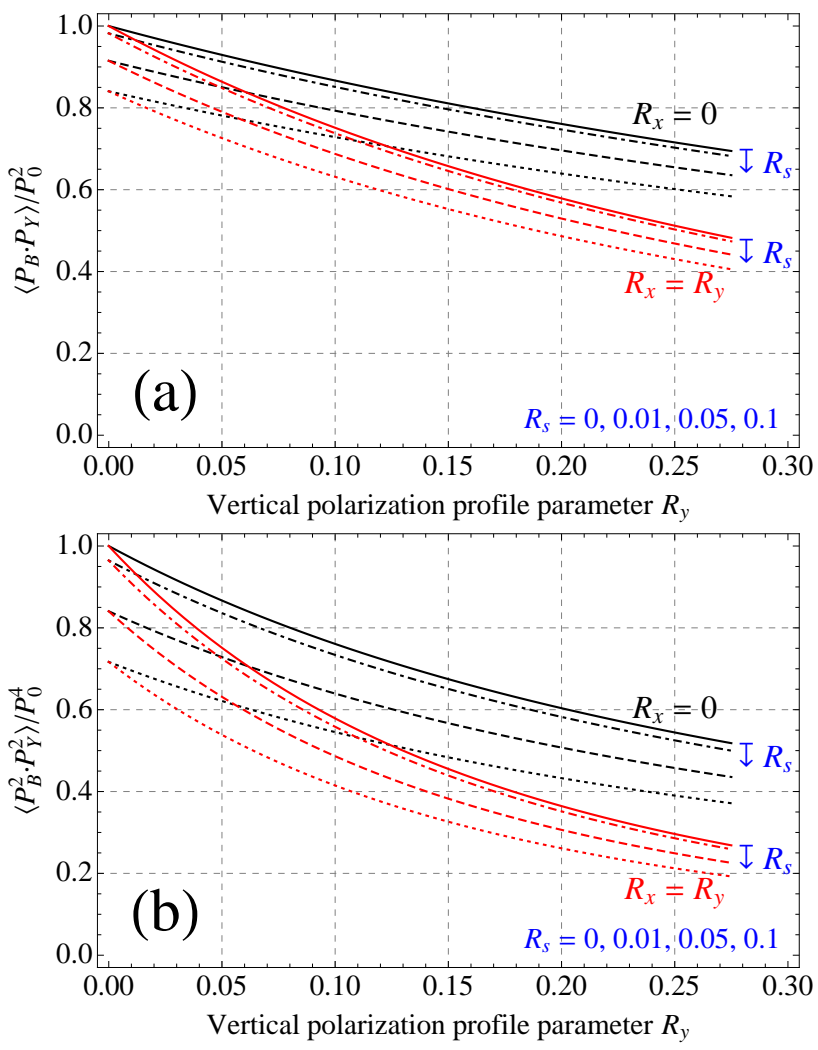

Figure 3: For double spin colliding beam experiments (a) relative reduction of the average polarization function $\left\langle P_{B} \cdot P_{Y}\right\rangle / P_{0}^{2}$, and (b) relative reduction of the figure of merit $\left\langle P_{B}^{2} \cdot P_{Y}^{2}\right\rangle / P_{0}^{4}$, as a function of the vertical profile parameter $R_{y}$. Assuming the same polarization profiles in both beams the cases $R_{x}=0$ and $R_{x}=R_{y}$ are shown for $R_{s}=0,0.01,0.05,0.1$ each. 